\title{
High resolution spectroscopy of stars with transiting planets ${ }^{\star}$
}

\section{The cases of OGLE-TR-10, 56, 111, 113, and TrES-1}

\author{
N. C. Santos ${ }^{1,2}$, F. Pont ${ }^{2}$, C. Melo ${ }^{3}$, G. Israelian ${ }^{4}$, F. Bouchy 5,6 , M. Mayor ${ }^{2}$, C. Moutou ${ }^{5}$, D. Queloz ${ }^{2}$, \\ S. Udry ${ }^{2}$, and T. Guillot ${ }^{7}$
}

\author{
${ }^{1}$ Centro de Astronomia e Astrofísica da Universidade de Lisboa, Observatório Astronómico de Lisboa, Tapada da Ajuda, \\ 1349-018 Lisboa, Portugal \\ e-mail: nuno@oal.ul.pt \\ 2 Observatoire de Genève, 51 Ch. des Maillettes, 1290 Sauverny, Switzerland \\ 3 European Southern Observatory, Casilla 19001, Santiago 19, Chile \\ ${ }^{4}$ Instituto de Astrofísica de Canarias, 38200 La Laguna, Tenerife, Spain \\ 5 Laboratoire d'Astrophysique de Marseille, Traverse du Siphon, 13013 Marseille, France \\ 6 Observatoire de Haute Provence, 04870 St. Michel l'Observatoire, France \\ 7 Observatoire de la Côte d'Azur, BP 4229, 06304 Nice Cedex 04, France
}

Received 24 November 2005 / Accepted 21 December 2005

\section{ABSTRACT}

Context. During the past years photometric surveys, later complemented by follow-up radial-velocity measurements, have revealed the presence of several new extra-solar transiting planets, in very short-period orbits. Many of the host stars are extremely faint $(V \sim 16)$, making high-precision spectroscopic measurements challenging.

Aims. We used the UVES spectrograph (VLT-UT2 telescope) to obtain high-resolution spectra of 5 stars hosting transiting planets, namely for OGLE-TR-10, 56, 111, 113, and TrES-1. The immediate objective is to derive accurate stellar parameters and chemical abundances.

Methods. The stellar parameters were derived from an LTE analysis of a set of Fe I and Fe II lines.

Results. Complementing the spectroscopic information with photometric transit curves and radial-velocity data from the literature, we then refined the stellar and planetary radii and masses. The obtained data were also used to study the relation between the stellar metallicity and orbital period of the planets.

Key words. stars: abundances - stars: fundamental parameters - planetary systems - planetary systems: formation

\section{Introduction}

Although the majority of the known extrasolar planets was discovered using the radial-velocity technique ${ }^{1}$, some major photometric transit searches are now delivering important results, giving new energy to the study of exoplanets. Many candidates have been announced by surveys like OGLE or TrES (Udalski et al. 2002a; Alonso et al. 2004), and the planetary nature was confirmed in a few cases by follow-up radial-velocity measurements (Konacki et al. 2003; Bouchy et al. 2004; Pont et al. 2004; Alonso et al. 2004; Bouchy et al. 2005a; Konacki et al. 2005). Together with the few transiting planets found in the context of radial-velocity surveys (Charbonneau et al. 2000; Henry et al. 2000; Sato et al. 2005; Bouchy et al. 2005b), these

\footnotetext{
* Based on observations collected at the ESO 8.2-m VLT-UT2 Kueyen telescope (program ID 75.C-0185).

${ }^{1}$ For a continuously updated list see table at http://obswww. unige.ch/Exoplanets
}

discoveries are now providing information about the physical properties of the giant planets themselves (e.g. radius, mean density) and opening up the possibility of comparing the observed properties with those predicted by the models.

The new detections have also raised a lot of scientific problems. Part of the discovered transiting planets (Konacki et al. 2003; Bouchy et al. 2004) have orbital periods of the order of 1-2 earth days. This is in clear contrast to the known pile-up of planets with periods above 3-days observed for (hot-jupiter) giant planets discovered by the radial-velocity technique (see e.g. Santos et al. 2005b). Whether this is simply the result of a detection bias or if this can be explained by some other physical process is now a matter of debate (e.g. Gaudi et al. 2005). On the other hand, among the confirmed transiting planets, HD 209458 (Charbonneau et al. 2000; Henry et al. 2000) has the lowest, and most anomalous mean density 
Table 1. Observations log.

\begin{tabular}{lccccr}
\hline \hline Star & Mag. $^{a}$ & $T_{\exp }[\mathrm{s}]$ & $N(\operatorname{Exp})$ & Bin & $S / N$ \\
\hline TrES-1 & 11.8 & 2000 & 1 & $1 \times 1$ & 160 \\
OGLE-TR-10 & 14.9 & 3000 & 4 & $2 \times 2$ & 110 \\
OGLE-TR-56 & 15.3 & 3000 & 5 & $2 \times 2$ & 100 \\
OGLE-TR-111 & 15.5 & 3000 & 6 & $2 \times 2$ & 90 \\
OGLE-TR-113 & 14.4 & 3000 & 3 & $2 \times 2$ & 115 \\
\hline
\end{tabular}

${ }^{a}$ Magnitudes are expressed in the $I$-band, except for TrES-1 (in V).

(Baraffe et al. 2005; Guillot \& Showman 2002). Curiously also, a relation between the planet mass and the orbital period also seems to exist (Mazeh et al. 2005). The understanding of these issues may give us some important clues about the processes of giant-planet formation and evolution.

In order to access these problems, we have to be able to derive accurate parameters for the planets. But the derivation of the planetary properties (mass, radius, and as a consequence their mean density) may depend considerably on the deduced parameters for the stellar host. For some cases, namely for the planets discovered in the context of the OGLE survey, the host stars are extremely faint $(V \sim 16)$, making it a difficult task to obtain precise spectroscopic parameters.

In this paper we present accurate stellar parameters and chemical abundances for 5 stars known to be orbited by transiting planets: OGLE-TR-10, OGLE-TR-56, OGLE-TR-111, and OGLE-TR-113, as well as for the brighter planet-host TrES-1. We use the obtained values to derive revised stellar and planetary radii and masses, as well as to study the relation between the presence of planets and the stellar metallicity (e.g. Gonzalez 1998; Santos et al. 2001, 2004) for short orbital period planets. In a separate paper we derive and discuss the ages of stars with short-period transiting planets (Melo et al. 2006).

\section{Observations}

The observations were carried out with the UVES spectrograph at the VLT-UT2 Kueyen telescope (program ID 75.C-0185) between April and May 2005 (service mode). For all stars, we opted for using a slit width of 0.9 arcsec, which provides a spectral resolution $R=\lambda / \Delta \lambda \sim 50000$. The observations were made using the Dichroic $390+580$ mode. The red portion of the spectra (used in the current paper) covers the wavelength domain between 4780 and $6805 \AA$, with a gap between 5730 and $5835 \AA$.

For the faint OGLE candidates ( $I$ magnitudes between 14.4 and 15.5), we made several exposures of 3000 seconds each. For each exposure, the CCD was read in $2 \times 2$ bins to reduce the readout noise and increase the number of counts in each bin. This procedure does not compromise the resolving power, since the sampling of the CCD is still higher (by a factor of 2) than the instrumental PSF. For the brighter TrES-1, we kept a $1 \times 1$ CCD binning. The total $S / N$ obtained for each star, as measured directly from small spectral windows with no clear spectral lines in the region near $6500 \AA$, is listed in Table 1.

For the OGLE stars, particular attention was paid to the orientation of the slit due to the relative crowdedness of the fields. The angle was chosen in each case using the images available
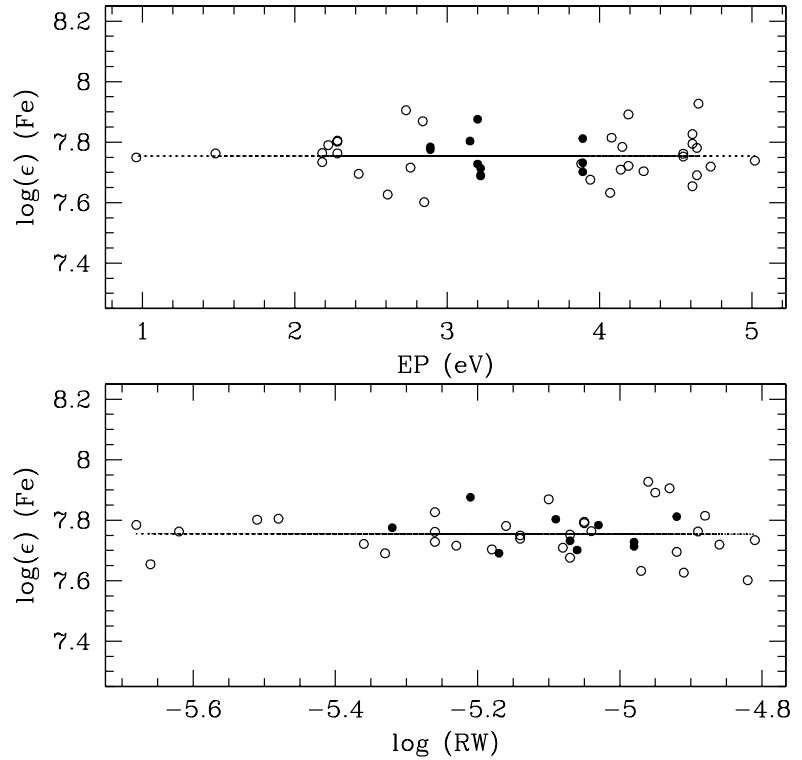

Fig. 1. Abundances given by individual Fe I (open circles) and Fe II (dots) lines as functions of the lower excitation potential (top) and the reduced equivalent width (bottom) for OGLE-TR-10. The lines represent least-square fits to the Fe I line data, and have a null slope. To derive the stellar parameters we iterated until the correlation coefficients between $\log \epsilon(\mathrm{Fe} \mathrm{I})$ and $\mathrm{EP}$ and between $\log \epsilon(\mathrm{Fe} \mathrm{I})$ and the reduced equivalent widths were zero, while the abundances derived from the $\mathrm{Fe}$ II lines were forced to be equal to those obtained from Fe I. For more details on the procedure see e.g. Santos et al. (2004).

at the OGLE website ${ }^{2}$, so that no other star was present in the UVES slit during the observation. For some of the targets, like OGLE-TR-10, this can be particularly important.

\section{Stellar parameters and metallicity}

Stellar parameters and chemical abundances were derived in LTE using the 2002 version of the code MOOG (Sneden 1973) ${ }^{3}$ and a grid of Kurucz Atlas plane-parallel model atmospheres (Kurucz 1993). The whole procedure is described in Santos et al. (2004, and references therein), and is based on analysis of $39 \mathrm{Fe} \mathrm{I}$ and $12 \mathrm{Fe}$ II weak lines, imposing excitation and ionization equilibrium (Fig. 1). As shown in Santos et al. (2004), this methodology gives excellent results for the derived stellar parameters of solar-type stars.

Line equivalent widths (EW) for the iron lines were measured using the IRAF" "splot" and "bplot" routines within the echelle package. The final derived stellar parameters and metallicities are presented in Table 2. In this table we also present the stellar parameters for the other stars hosting transiting planets but not studied in this paper. The error bars were derived as in Gonzalez \& Lambert (1996). In the error determination, the rms $(\sigma)$ around the average abundance given by

\footnotetext{
${ }^{2}$ http://www. astrouw. edu.pl/ ftp/ogle/index.html

${ }^{3}$ http://verdi.as.utexas.edu/moog.html

${ }^{4}$ IRAF is distributed by National Optical Astronomy Observatories, operated by the Association of Universities for Research in Astronomy, Inc., under contract with the National Science Foundation, USA.
} 
Table 2. Stellar parameters and metallicities for known transiting-planet host stars.

\begin{tabular}{lccccccl}
\hline \hline Star & $T_{\text {eff }}[\mathrm{K}]$ & $\log g(\mathrm{cgs})$ & $\xi_{\mathrm{t}}\left[\mathrm{km} \mathrm{s}^{-1}\right]$ & {$[\mathrm{Fe} / \mathrm{H}]$} & $N(\mathrm{Fe}$ I, Fe II $)$ & $\sigma(\mathrm{Fe}, \mathrm{Fe}$ II $)$ & Source \\
\hline TrES-1 & $5226 \pm 38$ & $4.40 \pm 0.10$ & $0.90 \pm 0.05$ & $0.06 \pm 0.05$ & 36,7 & $0.04,0.05$ & This paper \\
OGLE-TR-10 & $6075 \pm 86$ & $4.54 \pm 0.15$ & $1.45 \pm 0.14$ & $0.28 \pm 0.10$ & 33,11 & $0.08,0.06$ & This paper \\
OGLE-TR-56 & $6119 \pm 62$ & $4.21 \pm 0.19$ & $1.48 \pm 0.11$ & $0.25 \pm 0.08$ & 31,9 & $0.06,0.08$ & This paper \\
OGLE-TR-111 & $5044 \pm 83$ & $4.51 \pm 0.36$ & $1.14 \pm 0.10$ & $0.19 \pm 0.07$ & 31,7 & $0.07,0.18$ & This paper \\
OGLE-TR-113 & $4804 \pm 106$ & $4.52 \pm 0.26$ & $0.90 \pm 0.18$ & $0.15 \pm 0.10$ & 30,5 & $0.10,0.09$ & This paper \\
\hline OGLE-TR-132 & $6411 \pm 179$ & $4.86 \pm 0.14$ & $1.46 \pm 0.36$ & $0.43 \pm 0.18$ & - & - & Bouchy et al. (2004) \\
HD 149026 & $6147 \pm 50$ & $4.26 \pm 0.07$ & - & $0.36 \pm 0.05$ & - & - & Sato et al. (2005) \\
HD 189733 & $5050 \pm 50$ & $4.53 \pm 0.14$ & $0.95 \pm 0.07$ & $-0.03 \pm 0.04$ & - & - & Bouchy et al. (2005b) \\
HD 209458 & $6117 \pm 26$ & $4.48 \pm 0.08$ & $1.40 \pm 0.06$ & $0.02 \pm 0.03$ & - & - & Santos et al. (2004) \\
\hline
\end{tabular}

Table 3. Stellar parameters derived in previous studies for the 5 stars analyzed in the current paper.

\begin{tabular}{lcccl}
\hline \hline Star & $T_{\text {eff }}[\mathrm{K}]$ & $\log g(\mathrm{cgs})$ & {$[\mathrm{Fe} / \mathrm{H}]$} & Source \\
\hline TrES-1 & $5214 \pm 23$ & $4.52 \pm 0.05$ & $0.00 \pm 0.04$ & Laughlin et al. (2005) \\
& $5250 \pm 75$ & $4.6 \pm 0.2$ & $0.00 \pm 0.09$ & Sozzetti et al. (2004) \\
OGLE-TR-10 & $6220 \pm 140$ & $4.70 \pm 0.40$ & $0.39 \pm 0.14$ & Bouchy et al. (2005a) \\
& $5750 \pm 100$ & $4.4_{-0.9}^{+0.4}$ & $0.0 \pm 0.2$ & Konacki et al. (2005) \\
OGLE-TR-56 & $5970 \pm 150$ & $4.20 \pm 0.38$ & $0.17 \pm 0.19$ & Bouchy et al. (2005a) \\
& $\sim 5950$ & - & - & Konacki et al. (2003) \\
OGLE-TR-111 & $5070 \pm 400$ & $4.8 \pm 1.0$ & $0.12 \pm 0.28$ & Pont et al. (2004) \\
OGLE-TR-113 & $4800 \pm 150$ & $4.5_{-0.8}^{+0.5}$ & $0.0_{-0.3}^{+0.1}$ & Konacki et al. (2004) \\
& $4752 \pm 130$ & $4.50 \pm 0.53$ & $0.14 \pm 0.14$ & Bouchy et al. (2004) \\
\hline
\end{tabular}

the $\mathrm{Fe}$ I lines is used to compute the final uncertainties in the $[\mathrm{Fe} / \mathrm{H}]$ abundance, and not the $\sigma / \sqrt{n}$, with $n$ being the number of lines used. This may imply that the error bars are slighly overestimated.

\subsection{Comparison with the literature: the case of OGLE-TR-10}

The values presented in Table 2 for the 5 stars analyzed in the current paper are in excellent agreement with previous estimates from the literature (see Table 3 ). The main difference found is on the error bars of the measurements, which are much smaller in the current study. The only strong and important disagreement exists for OGLE-TR-10.

That star has been analyzed before by our team using an $S / N \sim 50$ FLAMES/UVES spectrum (Bouchy et al. 2005a), taken with the goal of obtaining precision radial-velocities for this star. We derived $T_{\text {eff }}=6220 \pm 140 \mathrm{~K}, \log g=4.70 \pm 0.40$, and $[\mathrm{Fe} / \mathrm{H}]=0.39 \pm 0.14$, values that are close (and compatible within the errors) to the ones derived in the current paper. In their paper on OGLE-TR-10, Konacki et al. (2005) derive a clearly different set of stellar parameters for this star using a low $S / N=44$ spectrum. They have used a spectral fitting procedure to derive the effective temperature and obtained $\left(T_{\text {eff }}\right.$, $\log g,[\mathrm{Fe} / \mathrm{H}])=(5750 \mathrm{~K}, 4.4 \mathrm{dex}, 0.0 \mathrm{dex})$.

The lower $T_{\text {eff }}$ value (by $325 \mathrm{~K}$ ) derived by Konacki et al. (2005) can now be reasonably discarded on the basis of our analysis. The difference between their value and the one derived in the current paper can be due to several effects, including the different spectroscopic analysis methods used, the poorer quality of the spectrum taken by Konacki et al. (2005), or to some other problem related e.g. to the existence of stellar blends (some fainter stars exist in the proximity of OGLE-TR-10 - see Sect. 2). At this moment it is difficult to understand which, if any, of these possibilities is the cause for the observed discrepancy. Other stars analyzed by both teams (e.g. OGLE-TR-113) do not show such a strong difference in the derived stellar parameters.

\subsection{Using $H_{\alpha}$ to check the temperatures}

In order to check the excitation temperatures derived from the previous spectroscopic analysis, we compared the observed $\mathrm{H}_{\alpha}$ line profiles with synthetic profiles for metal-rich dwarfs $([\mathrm{M} / \mathrm{H}]=0.3$ and $\log g=4.5)$ computed by R. Kurucz ${ }^{5}$ for different temperatures. The results of this comparison can be seen in Fig. 2 and they show that the $\mathrm{H}_{\alpha}$ temperatures agree with the values derived in the previous section for the OGLE stars.

It is known that the analysis of $\mathrm{H}_{\alpha}$ profiles can provide accurate estimates for the effective temperatures of solar-type dwarfs. The wings of these lines are very sensitive to $T_{\text {eff }}$ changes, while relatively insensitive to surface gravity and metallicity variations (e.g. Fuhrmann et al. 1993; Barklem et al. 2002). However, the fitting of $\mathrm{H}_{\alpha}$ profiles can be subject to several sources of error. Given the large width of the line, important uncertainties may come from the normalization and

\footnotetext{
5 These can be found at

http: //kurucz.harvard.edu/grids.html
} 

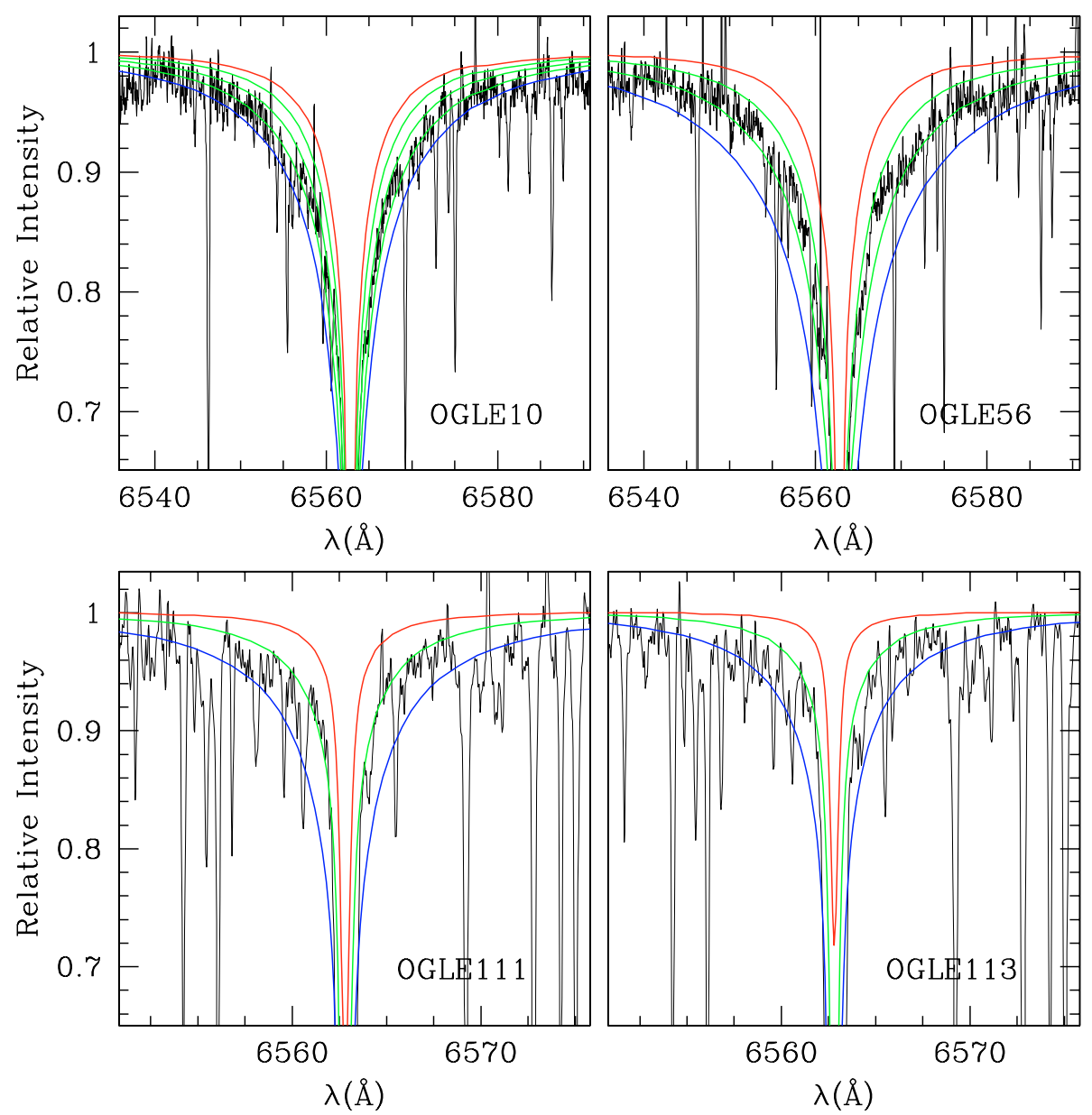

6580

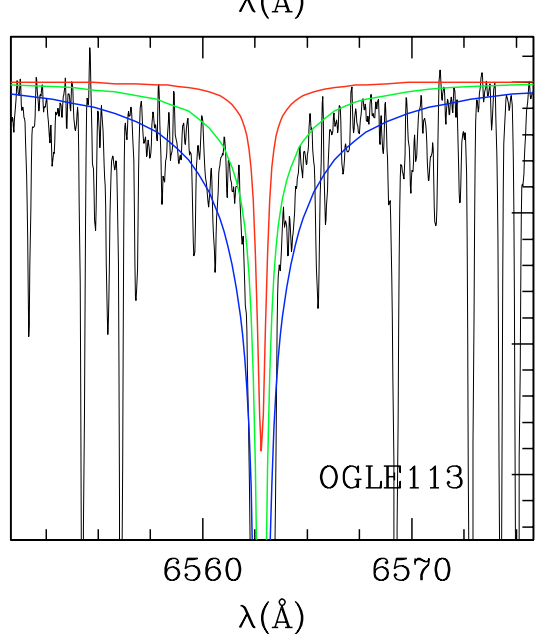

Fig. 2. Observed $\mathrm{H}_{\alpha}$ profiles for OGLE-TR-10, 56, 111 and 113, together with spectral synthesis for different temperatures, log $g=4.5$ and $[\mathrm{M} / \mathrm{H}]=0.3$. For OGLE-TR-10, 5 synthesis are shown, for temperatures of (from top to bottom) 5500, 5750, 6000, 6250 and 6500 K. For OGLE-TR-111 and 113 three synthesis are presented, corresponding to temperatures of $(4500 \mathrm{~K}, 5000 \mathrm{~K}, 5500 \mathrm{~K})$ and $(4250 \mathrm{~K}, 4750 \mathrm{~K}$, $5250 \mathrm{~K}$ ), respectively. For OGLE-TR-56 we present four synthesis, corresponding to temperatures of $5500 \mathrm{~K}, 6000 \mathrm{~K}, 6500 \mathrm{~K}$ and $7000 \mathrm{~K}$.

continuum determination, in particular when dealing with relatively low- $S / N$ echelle spectra where one single spectral order spans only a few dozen Angstroms. Furthermore, the strong line blending present in the spectra of metal-rich dwarfs makes the $\mathrm{H}_{\alpha}$ line profile determination quite uncertain. These problems may be at the origin of the very different effective temperature derived by Konacki et al. (2005) for OGLE-TR-10. In this paper, $\mathrm{H}_{\alpha}$ profiles are thus presented only as a confirmation of our Fe-line based temperatures.

\section{The metallicity of stars with short-period planets}

The results presented in Table 2 show that the stars hosting transiting planets that were studied in the current paper, and in particular those found by transit surveys like OGLE, follow the general trend observed for stars with giant planets: they are metal-rich in comparison with the Sun (Gonzalez 1998; Gonzalez et al. 2001; Santos et al. 2001, 2004, 2005a; Fischer \& Valenti 2005).

The stars hosting the three shortest period planets listed in the table also follow this trend: OGLE-TR-56 and 113, analyzed in the current paper, and OGLE-TR-132, with a less reliable $[\mathrm{Fe} / \mathrm{H}]$ value, all with orbital periods shorter than 2-days. But although they do not present metallicities that are significantly above the rest of the sample, a small "excess" cannot be excluded. For instance, the lower metallicity amid the stars with planets in orbital periods below 2 days is +0.15 (OGLE-TR-113), while this value decreases to -0.03 (HD 189733) in the orbital period range from 2-3 days.

In order to check if some metallicity trend exists among the stars with short-period planets, we used the spectroscopic data from Table 2, together with the metallicity values listed in Santos et al. (2004) and Santos et al. (2005a), as well as the orbital periods listed on the Geneva web page ${ }^{6}$, to derive the average $[\mathrm{Fe} / \mathrm{H}]$ of stars with planets in bins of orbital period. The results show that stars with planets having orbital periods below 2 days (OGLE-TR-56, 113 and 132) have an average metallicity of +0.28 ( $\sigma=0.14 \mathrm{dex}, 3$ stars $)$; excluding OGLE-TR-132 this value would decrease to +0.20 . This average metallicity decreases to $+0.23(\sigma=0.14)$ in the period interval between 2 and 3 days ( 7 stars), to +0.21 ( $\sigma=0.11$ ) for periods between 3 and 5 days (17 stars), and to +0.19

\footnotetext{
${ }^{6}$ http://obswww.unige.ch/Exoplanets
} 
$(\sigma=0.22)$ for periods between 5 and 10 days $(5 \mathrm{stars})^{7}$. Note that here we have excluded the M-dwarf planet-hosts, as their metallicities are less reliable.

Particular care must be taken when looking at these results. First of all, the number of stars is statistically small. Furthermore, a significant number of them come from different galactic regions and populations. The OGLE objects are faint and distant stars (at a few Kpc from the Sun) seen in the direction of the Galactic bulge (OGLE-TR-10 and 56) and Carina arm (OGLE-TR-111, 113 and 132). Given the radial metallicity gradient in the Galaxy (Nordström et al. 2004), they may belong to different populations when compared to the solarneighborhood field stars studied in radial-velocity surveys (see discussion in Sozzetti 2004). For instance, the average metallicity of the OGLE stars (that span a large range of effective temperatures and are orbited by planets with periods from $\sim 1.2$ to $\sim 4$ days) is +0.26 dex, clearly above the average value for the other 4 stars in Table $2(+0.10)$.

It has been suggested that stars orbited by short-period planets (hot- and very-hot-jupiters) may have an average metallicity above the one found for other planet hosts (Gonzalez 1998; Queloz et al. 2000; Santos et al. 2003; Sozzetti 2004; Fischer \& Valenti 2005). As seen above, whether this trend is real or even intensified for the very-hot-jupiters is not clear, but if this were the case, several possible explanations exist.

The possibility that planet migration is somewhat controlled by the dust content of the disk seems to be reasonably ruled out by current models (Livio \& Pringle 2003), which usually predict that migration rates are not strongly affected by the dust content of the gas disk. On the other hand, although migration induced by interactions with the disk of planetesimals is possible, it seems to require a very high value for the mass of the planetesimal disk (e.g. Murray et al. 1998).

Another possibility is that a more significant migration could increase planetesimal scattering into the star and therefore pollute the stellar outer layers. This would lead to an increase in the observed stellar iron-to-hydrogen ratio (e.g. Murray et al. 2001). The lack of a general correlation between the depth of the convective zone (or alternatively the effective temperature) and the metallicity of the star implies that this possibility is unlikely (Pinsonneault et al. 2001; Santos et al. 2003; Fischer \& Valenti 2005), although probably not completely excluded (Vauclair 2004). We should add that we do find a weak correlation between effective temperature and stellar metallicity in stars with short period planets $(P<10$ days). However, not only is this correlation not significant, but it may also simply reflect biases in the samples like the cut in $B-V$ of the radial-velocity planet-search samples (e.g. Santos et al. 2004) and/or the galactic chemical evolution gradient (Edvardsson et al. 1993).

An alternative explanation for the slight metallicity-period trend could have to do with planetary internal structure arguments. If a planet forming in a higher metallicity disk acquires a more massive core or has a higher metal-to-hydrogen

7 The pile-up of planets in the orbital period regime around 3-5 days has been thoroughtly discussed in the literature (e.g. Udry et al. 2003; Gaudi et al. 2005). ratio (e.g. Pollack et al. 1996; Ida \& Lin 2004; Guillot et al. 2006), its higher density will help its survival against evaporation (Baraffe et al. 2004; Lecavelier des Etangs et al. 2004). At least in one case evidence was found for a hot-jupiter having a core mass close to $70 M_{\oplus}$ and orbiting a very metal-rich star (Sato et al. 2005). This idea could, in principle, also explain any possible metallicity-effective temperature correlation, if one considers that only the most "metal-rich" planets would survive close to a higher temperature dwarf emitting a higher UV-flux.

Finally, such a correlation may arise indirectly from the combination of a faster growth of planets and their migration. This occurs in core-accretion models in which the loss of the gas disk occurs on timescales similar to the growth of the planets. In that case, a more rapid growth in a metal-rich environment will imply a more pronounced migration (see e.g. Lecar \& Sasselov 2003; Ida \& Lin 2004; Alibert et al. 2005; Guillot $\&$ Hueso 2006).

\section{Revised planetary masses and radii}

Using the stellar parameters presented in Table 2, we have recalculated the planetary and stellar radii and masses following to the method described in Bouchy et al. (2005a). The constraints from the spectroscopic parameters and the photometric transit shape are combined by $\chi^{2}$ minimization to derive the mass and radius of the host star under the assumption that it is a main-sequence star of any age, as described by the Girardi et al. (2000) stellar evolution models. The radii of the planets are obtained from the radius ratio given by the light curve, and the planet's mass is derived from the mass function given by the radial velocity orbit.

In this procedure we adopted the latest and best observations known to us for the light curves and radial velocity orbits (see Table 4 for references). For OGLE-TR-111, we also used the new ephemerids found by the OGLE team from recent observations: $T_{\text {tr }}=2452330.46228, P=4.014442$ days (Udalski, priv. comm.). For OGLE-TR-10 we used both the original photometry from the OGLE campaign and the new results from Holman et al. (2005).

Table 4 gives the masses and radii values for host stars and planets derived from our analysis. The error bars do not include the systematic uncertainties on the host star properties due to the uncertainties on stellar evolution models, bolometric corrections, and atmosphere models. Given the similarity of stellar parameters derived in this paper to those found in the literature for OGLE-TR-113 and TrES-1, we did not derive revised parameters for these two stars.

In Fig. 3 we plot a mass-radius diagram for the known transiting planets. Without a detailed evolution model accounting for the planet's mass, age, and stellar insolation, one cannot infer the composition of the planets directly from such a diagram (e.g. Guillot 2005). However, as also previously noted in different works (e.g. Baraffe et al. 2005; Laughlin et al. 2005), both HD 209458b and OGLE-TR-10b (considering the original result by Bouchy et al. 2005a) appear to have anomalous mean densities. For this case the use of the recent photometry by Holman et al. (2005), together with the stellar parameters 
Table 4. Derived stellar and planetary radii and masses for stars with transiting planets.

\begin{tabular}{|c|c|c|c|c|c|c|}
\hline Star & $\begin{array}{l}M_{\text {star }} \\
{\left[M_{\odot}\right]} \\
\end{array}$ & $\begin{array}{l}R_{\text {star }} \\
{\left[R_{\odot}\right]}\end{array}$ & $\begin{array}{c}m_{\mathrm{pl}} \\
{\left[M_{\text {Jup }}\right]}\end{array}$ & $\begin{array}{c}r_{\mathrm{pl}} \\
{\left[R_{\mathrm{Jup}}\right]}\end{array}$ & $\begin{array}{l}\text { Reference for } \\
\text { light curve }\end{array}$ & $\begin{array}{l}\text { Reference for } \\
\text { radial velocity }\end{array}$ \\
\hline OGLE-TR-10 & $1.17 \pm 0.04$ & $14 \pm 0.05$ & $.63 \pm 0.14$ & $1.14 \pm 0.09$ & Holman et al. ( & $\begin{array}{l}05 a) \\
05)\end{array}$ \\
\hline OGLE- & $117+0.04$ & \pm 0.05 & 063 & 0 & 2002c) & $\begin{array}{l}\text { y et al. (2005a) } \\
\text { ki et al. (2005) }\end{array}$ \\
\hline OGLE-TR-56 & $7 \pm 0.04$ & $5 \pm 0.06$ & 0.13 & 08 & 2002c) & $\begin{array}{l}\text { ki et al. (2003) } \\
\text { et al. (2004) } \\
\text { y et al. (2005a) }\end{array}$ \\
\hline OGLE-TR-111 & $0.81 \pm 0.02$ & $0.83 \pm 0.02$ & $0.52 \pm 0.13$ & $0.97 \pm 0.06$ & Udalski et al. (2002b) & Pont et al. (2004) \\
\hline
\end{tabular}

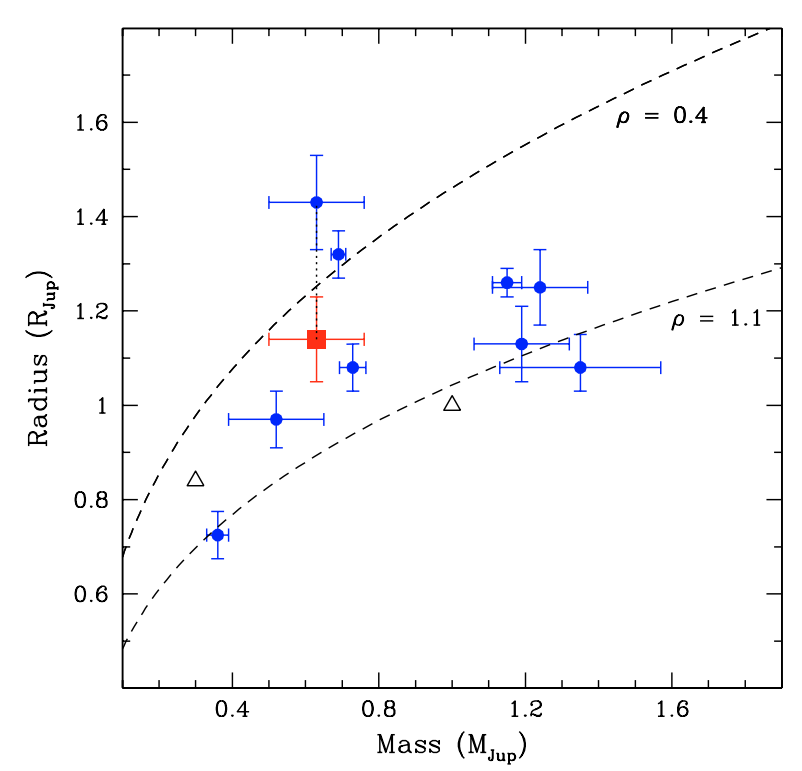

Fig. 3. Mass-radius diagram for the known transiting planets. For OGLE-TR-10b we show both values for the radius listed in Table 4, the filled square denoting the values derived using the unpublished photometry by Holman et al. (2005). A dotted line connects the two different points of OGLE-TR-10b. Two iso-density curves are also shown for mean densities of 0.4 and $1.1 \mathrm{~g} \mathrm{~cm}^{-3}$. The open triangles denote the positions of Jupiter and Saturn. The data were taken from Table 4 for OGLE-TR-10b, 56b and $111 \mathrm{~b}$, and from Laughlin et al. (2005, for TrES-1b), Bouchy et al. (2004, OGLE-TR-113b), Moutou et al. (2004, OGLE-TR-132b), Sato et al. (2005, HD 149026b), Bouchy et al. (2005b, HD 189733b), Laughlin et al. (2005) and Brown et al. (2001) (HD 209458b).

derived in the current paper, seem to place this planet at a rather normal position on this diagram.

Specific evolution models (Guillot et al., in prep.) indicate that, although it appears relatively "normal" in Fig. 3, HD 189733 b is also anomalously large. This is due to its relatively large mass and low insolation that yield evolution models to predict a smaller radius.

\section{Concluding remarks}

We used high resolution and $S / N$ spectra taken with the UVES spectrograph (VLT-UT2) to derive accurate stellar parameters and metallicities for 5 stars known to be transited by giant planets. The resulting parameters were used to study both the relation between the stellar metallicity and the existence of giant planets in short period-orbits and to derive revised masses and radii for the transiting planets.

The results show that the stars with transiting planets studied in the current paper follow the general trend of high metallicities found for the majority of the planet hosts (Gonzalez 1998; Santos et al. 2001). A weak correlation is found for the average metallicity as a function of the orbital period among the short period planets. The addition of more data is needed to confirm or deny this result.

Acknowledgements. We would like to thank our referee, A. Sozzetti, for the useful comments and suggestions. Support from the Fundação para a Ciência e a Tecnologia (Portugal) to N.C.S. in the form of a scholarship (reference SFRH/BPD/8116/2002) and a grant (reference POCI/CTE-AST/56453/2004) is gratefully acknowledged.

\section{References}

Alibert, Y., Mordasini, C., Benz, W., \& Winisdoerffer, C. 2005, A\&A, 434, 343

Alonso, R., Brown, T. M., Torres, G., et al. 2004, ApJ, 613, L153

Baraffe, I., Chabrier, G., Barman, T. S., et al. 2005, A\&A, 436, L47

Baraffe, I., Selsis, F., Chabrier, G., et al. 2004, A\&A, 419, L13

Barklem, P. S., Stempels, H. C., Allende Prieto, C., et al. 2002, A\&A, 385,951

Bouchy, F., Pont, F., Santos, N. C., et al. 2004, A\&A, 421, L13

Bouchy, F., Pont, F., Melo, C., et al. 2005a, A\&A, 431, 1105

Bouchy, F., Udry, S., Mayor, M., et al. 2005b, A\&A, 444, L15

Brown, T. M., Charbonneau, D., Gilliland, R. L., Noyes, R. W., \& Burrows, A. 2001, ApJ, 552, 699

Charbonneau, D., Brown, T., Latham, D., \& Mayor, M. 2000, ApJ, 529, L45

Edvardsson, B., Andersen, J., Gustafsson, B., et al. 1993, A\&A, 275, 101

Fischer, D. A., \& Valenti, J. 2005, ApJ, 622, 1102

Fuhrmann, K., Axer, M., \& Gehren, T. 1993, A\&A, 271, 451

Gaudi, B. S., Seager, S., \& Mallen-Ornelas, G. 2005, ApJ, 623, 472

Girardi, L., Bressan, A., Bertelli, G., \& Chiosi, C. 2000, A\&AS, 141, 371

Gonzalez, G. 1998, A\&A, 334, 221

Gonzalez, G., \& Lambert, D. L. 1996, AJ, 111, 424

Gonzalez, G., Laws, C., Tyagi, S., \& Reddy, B. E. 2001, AJ, 121, 432

Guillot, T. 2005, Ann. Rev. Earth Planet. Sci., 33, 493

Guillot, T., \& Hueso, R. 2006, MNRAS, submitted

Guillot, T., Santos, N. C., Pont, F., et al. 2006, Nature, submitted

Guillot, T., \& Showman, A. P. 2002, A\&A, 385, 156 
Henry, G. W., Marcy, G. W., Butler, R. P., \& Vogt, S. S. 2000, ApJ, 529, L41

Holman, M. J., Winn, J. N., Stanek, K. Z., et al. 2005, ArXiv Astrophysics e-prints [arXiv: astro-ph/0506569]

Ida, S., \& Lin, D. N. C. 2004, ApJ, 616, 567

Konacki, M., Torres, G., Jha, S., \& Sasselov, D. 2003, Nature, 421, 507

Konacki, M., Torres, G., Sasselov, D. D., \& Jha, S. 2005, ApJ, 624, 372

Konacki, M., Torres, G., Sasselov, D. D., et al. 2004, ApJ, 609, L37

Kurucz, R. 1993, ATLAS9 Stellar Atmosphere Programs and $2 \mathrm{~km} \mathrm{~s}^{-1}$ grid, Kurucz CD-ROM No. 13 (Cambridge, Mass.: Smithsonian Astrophysical Observatory)

Laughlin, G., Wolf, A., Vanmunster, T., et al. 2005, ApJ, 621, 1072

Lecar, M., \& Sasselov, D. D. 2003, ApJ, 596, L99

Lecavelier des Etangs, A., Vidal-Madjar, A., McConnell, J. C., \& Hébrard, G. 2004, A\&A, 418, L1

Livio, M., \& Pringle, J. E. 2003, MNRAS, 346, L42

Mazeh, T., Zucker, S., \& Pont, F. 2005, MNRAS, 356, 955

Melo, C., Santos, N., Israelian, G., et al. 2006, A\&A, submitted

Moutou, C., Pont, F., Bouchy, F., \& Mayor, M. 2004, A\&A, 424, L31

Murray, N., Hansen, B., Holman, M., \& Tremaine, S. 1998, Science, 279, 69

Murray, N., Chaboyer, B., Arras, P., Hansen, B., \& Noyes, R. W. 2001, ApJ, 555, 801
Nordström, B., Mayor, M., Andersen, J., et al. 2004, A\&A, 418, 989 Pinsonneault, M. H., DePoy, D. L., \& Coffee, M. 2001, ApJ, 556, L59 Pollack, J., Hubickyj, O., Bodenheimer, P., et al. 1996, Icarus, 124, 62 Pont, F., Bouchy, F., Queloz, D., et al. 2004, A\&A, 426, L15 Queloz, D., Mayor, M., Weber, L., et al. 2000, A\&A, 354, 99 Santos, N. C., Israelian, G., \& Mayor, M. 2001, A\&A, 373, 1019 Santos, N. C., Israelian, G., Mayor, M., Rebolo, R., \& Udry, S. 2003 , A\&A, 398, 363

Santos, N. C., Israelian, G., \& Mayor, M. 2004, A\&A, 415, 1153

Santos, N. C., Israelian, G., Mayor, M., et al. 2005a, A\&A, 437, 1127

Santos, N. C., Mayor, M., \& Benz, W. 2005b, Science, 310, 251

Sato, B., Fischer, D. A., Henry, G. W., et al. 2005, ApJ, 633, 465

Sneden, C. 1973, Ph.D. Thesis, Univ. of Texas

Sozzetti, A. 2004, MNRAS, 354, 1194

Sozzetti, A., Yong, D., Torres, G., et al. 2004, ApJ, 616, L167

Torres, G., Konacki, M., Sasselov, D. D., \& Jha, S. 2004, ApJ, 609, 1071

Udalski, A., Paczynski, B., Zebrun, K., et al. 2002a, Acta Astron., 52, 1

Udalski, A., Szewczyk, O., Zebrun, K., et al. 2002b, Acta Astron., 52, 317

Udalski, A., Szymanski, M., Kubiak, M., et al. 2002c, Acta Astron., 52, 217

Udry, S., Mayor, M., \& Santos, N. 2003, A\&A, 407, 369

Vauclair, S. 2004, ApJ, 605, 874 